\title{
ARTICLE Class-switch recombination to IgA in the Peyer's patches requires natural thymus-derived Tregs and appears to be antigen independent
}

\author{
Inta Gribonika ${ }^{1}$, Dubravka Grdic Eliasson ${ }^{1}$, Rakesh K. Chandode ${ }^{1}$, Karin Schön ${ }^{1}$, Anneli Strömberg ${ }^{1}$, Mats Bemark ${ }^{1,2}$ and Nils Y. Lycke ${ }^{1}$
}

\begin{abstract}
Our understanding of how class-switch recombination (CSR) to IgA occurs in the gut is still incomplete. Earlier studies have indicated that Tregs are important for IgA CSR and these cells were thought to transform into follicular helper T cells (Tfh), responsible for germinal center formation in the Peyer's patches (PP). Following adoptive transfer of T-cell receptor-transgenic (TCRTg) CD4 T cells into nude mice, we unexpectedly found that oral immunization did not require an adjuvant to induce strong gut lgA and systemic lgG responses, suggesting an altered regulatory environment in the PP. After sorting of splenic TCR-Tg CD4 T cells into $\mathrm{CD} 25^{+}$or $\mathrm{CD} 25^{-}$cells we observed that none of these fractions supported a gut IgA response, while lgG responses were unperturbed in mice receiving the $\mathrm{CD} 25^{-}$cell fraction. Hence, while Tfh functions resided in the $\mathrm{CD} 25^{-}$fraction the IgA CSR function in the PP was dependent on $\mathrm{CD} 25^{+}$Foxp $^{+}$Tregs, which were found to be Helios ${ }^{+}$neuropilin- $1^{+}$thymus-derived Tregs. This is the first study to demonstrate that Tfh and IgA CSR functions are indeed, unique, and separate functions in the PP with the former being TCR-dependent while the latter appeared to be antigen independent.
\end{abstract}

Mucosal Immunology (2019) 12:1268-1279; https://doi.org/10.1038/s41385-019-0202-0

\section{INTRODUCTION}

The most important inductive sites for gut lgA B-cell responses are the Peyer's patches (PP) of the small intestine. ${ }^{1-3}$ Although significant efforts have been devoted to understanding how classswitch recombination (CSR) to $\lg A$ in the PP is regulated many questions still remain. In the early studies researchers identified the presence of "IgA-switch T cells" that acted on IgM B cells to undergo CSR to $\lg A$ in the PP, but their findings also suggested that these cells could not support terminal differentiation of IgAswitched B cells. ${ }^{4}$ More recent studies have demonstrated that regulatory $T$ cells (Tregs) are important for IgA CSR, but Th17 cells have also been implicated in gut IgA responses. ${ }^{5-7}$ In fact, sorted Tregs exhibited plasticity upon adoptive transfer and differentiated into $\mathrm{T}$ follicular helper cells (Tfh), which were assumed to regulate IgA CSR in the PP germinal centers (GC). ${ }^{8}$ Tregs also promoted the formation of $\lg A$-producing plasma cells in a colitis model and Treg-depletion resulted in reduced gut IgA production, linking Tregs to $\lg A \mathrm{CSR}^{5,9,10}$ On the other hand, Th17 cells acquired a Tfh phenotype in the PP after adoptive transfer into Tcell receptor a-deficient $\left(\mathrm{TCRa}^{-1-}\right)$ mice and induced IgAproducing GC. ${ }^{6}$ These authors could also show that a chimeric $\mathrm{TCRa}^{-1-}$ mouse reconstituted with bone marrow from Rorytdeficient $\left(\right.$ Rorc $\left.^{-1-}\right)$ mice largely failed to respond with specific gut $\lg A$ antibody production to oral immunizations with cholera toxin (CT), suggesting a need for Th17 cells for IgA CSR. ${ }^{6}$ Thus, both Tregs and Th17 cells exhibited extensive plasticity in the PP and appeared to converge into Tfh cells, but whether these Th cells directly induced IgA CSR in activated $B$ cells remained to be shown.
TGF $\beta$ is the main switch factor for IgA CSR as demonstrated in TGF $\beta 1$ - and in TGF $\beta \mathrm{RII}^{-1-}$ mice. ${ }^{11-13}$ Tregs are known to produce TGF $\beta$, but evidence in support of production of TGF $\beta$ by Th17 cells is lacking. ${ }^{14}$ Rather Th17 cells are known to produce IL-21, which has been ascribed an IgA CSR effect, albeit this appears to be relatively minor to that of TGF $\beta .^{15}$ Hence, classical Th17 cells are not an obvious candidate for driving IgA CSR, but could acquire a Tfh function and influence $\operatorname{lgA}$ responses in several ways. ${ }^{16,17}$ For example, strong evidence supports that IL-17 can influence IgA transport through upregulation of the polylg-receptor on epithelial cells and IL-21 is a critical factor for IgA B cell differentiation in the GC. ${ }^{18}$

Whereas Tregs can be separated into thymic-derived natural Tregs (tTregs), with recognition of endogenous antigens, or peripherally induced Tregs, (pTregs), recognizing foreign antigens, both subsets can produce TGF $\beta$ and both are found in the PPs. ${ }^{719,20}$ Because both these populations are identifiable as $\mathrm{CD}_{25}{ }^{+} \mathrm{Foxp}^{+} \mathrm{CD}^{+} \mathrm{T}$ cells, no dissociation of these subsets for TGF $\beta$-driven IgA CSR was presented in previous publications. For example, in vitro studies have shown that $\mathrm{CD} 25^{+} \mathrm{Foxp}^{+} \mathrm{CD}^{+} \mathrm{T}-$ cells induce splenic $B$ cells to produce $\lg A .{ }^{21}$ Besides, multiple other TGF $\beta$ producing cells in, or close to, the PP have been reported, such as subsets of dendritic cells (DC) and epithelial cells, which both could contribute to IgA CSR. ${ }^{14}$ Also B-cell intrinsic production of TGF $\beta 1$ has been found to play a role for $\lg A B$ responses, primarily under T-cell-independent conditions. ${ }^{22}$ Hence, the cellular source for TGF $\beta$ in the PP is not clear. In addition, the production of TGF $\beta$ itself is in a latent form and, therefore, other cells may be needed to activate latent TGF $\beta$. This

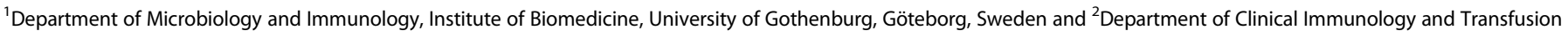
Medicine, Sahlgrenska University Hospital, Gothenburg, Sweden

Correspondence: Nils Y. Lycke (nils.lycke@microbio.gu.se)

Received: 23 April 2019 Revised: 5 August 2019 Accepted: 15 August 2019

Published online: 9 September 2019 
was demonstrated for a DC subset in the subepithelial dome (SED) of the PP, that expressed av $\beta 8$ integrin, which can activate latent TGF $\beta .{ }^{23,24}$ Also Tregs and in particular tTregs express av $\beta 8$ integrin and can activate latent TGF $\beta{ }^{24}$ Furthermore, $M$ cells of the follicle associated epithelium (FAE) that sample luminal antigens and mesenchymal cells, that influence $M$ cells through RANKLexpression, have been implicated in driving gut $\operatorname{lgA} B$-cell responses. ${ }^{25,26}$ Also cells producing a proliferation inducing ligand (APRIL), B-cell activating factor or retinoic acid, such as DCs, can influence IgA CSR or promote already IgA-committed B cells. ${ }^{27-29}$ Thus, although many cell types have been implicated as regulators of IgA CSR, it is still unclear how IgA responses in the gut are regulated and what the cellular source is of the TGF $\beta$.

The present study was undertaken to investigate in detail the CD4 T-cell requirements for gut IgA responses, with special focus on CD4 T-cell subsets needed to generate IgA plasma cells in the lamina propria (LP) of the small intestine. ${ }^{30}$ To this end we adoptively transferred CD4 T cells from ovalbumin (OVA)-specific T-cell receptor-transgenic (TCR-Tg) mice into nude recipient mice (nu/nu) that were subsequently orally immunized with OVA in the absence or presence of $C T$ adjuvant. We provide compelling evidence that $\lg A$ responses in the PP requires two distinct and separate CD4 T-cell subsets, tTregs and Tfh, to induce IgA CSR and terminal IgA B-cell differentiation, respectively. The former is unrelated to the antigen and independent of the microbiota, while the latter recognizes antigen and could be dependent on the microbiota.

\section{RESULTS}

A mouse model for oral immunization not requiring adjuvant We made an unexpected observation following oral immunization with antigen alone in DO11.10 mice (BALB/c TCR-Tg for OVA peptide 323). ${ }^{31,32}$ As it is commonly known that oral immunizations with soluble protein antigens require an effective adjuvant to be successful, we were surprised to find that similar strong levels of IgA plasma cell responses in the gut LP were achieved with or without the gold-standard oral CT adjuvant (Fig. 1a). ${ }^{33,34}$ The small intestinal IgA spot-forming cell (SFC) response to OVA was indistinguishable between the two groups, while the antiOVA IgA SFC LP response in BALB/c wild-type (WT) mice was hardly detectable in the absence of CT-adjuvant (Fig. 1a). By contrast, parenteral immunizations (intraperitoneal (i.p)) in the DO11.10 mice required CT adjuvant to give substantial splenic lgG SFC responses and, indeed, the response in DO11.10 mice mimicked that observed in WT mice, i.e., responses were dramatically augmented by adjuvant (Fig. 1a). Thus, we concluded that the regulatory environment in the gut-associated lymphoid tissues (GALT) of D011.10 mice appeared to differ from that prevailing in WT mice or that found at the systemic level.

To focus on the regulatory T-cell environment in the GALT of D011.10 mice, we employed an adoptive transfer model using $\mathrm{BALB} / \mathrm{c}$ nu/nu mice, lacking all thymus-dependent T cells. ${ }^{35}$ Following the transfer of splenic DO11.10 CD4 T cells and three oral immunizations with OVA, with or without $C T$ adjuvant, we found again that irrespective of CT adjuvant we observed substantial and comparable gut IgA LP anti-OVA SFC responses, while i.p immunizations required $C T$ adjuvant to stimulate significant $\lg G$ SFC responses in the spleen (Fig. 1b). These IgA and IgG SFC responses were strictly T-cell dependent (TD) as no response was seen in BALB/c nu/nu mice without CD4 T-cell transfer (Fig. 1b). As oral treatment with OVA without adjuvant is also known to induce tolerance, we investigated if we had induced immune suppression at the systemic level. ${ }^{36,37}$ Indeed, we found that oral tolerance in DO11.10 mice, evaluated as a footpad DTH-reaction, was comparable to that achieved in WT mice, suggesting that immune regulation at the systemic level in our model was similar to that observed in WT mice (Fig. 1c). ${ }^{38,39}$ Hence, the regulatory CD4 T-cell environment in the GALT of D011.10 mice appeared distinctly different from that observed in WT mice, whereas regulation at the systemic level was comparable between the two strains.

Whereas PPs are the primary site for induction of $\lg A$ responses following oral immunizations in WT mice, we asked whether this was also the case in nu/nu mice after adoptive transfer of CD4 T cells. ${ }^{3}$ For these experiments, we used OTII OVAp323-specific TCR-Tg mice and adoptive transfer of splenic CD4 T cells into WT or $\mathrm{C} 57 \mathrm{BI} / 6 \mathrm{nu} / \mathrm{nu}$ mice. ${ }^{32}$ Following oral immunizations with OVA in nu/nu mice, we again observed comparable levels of gut LP IgA SFC, irrespective of if we had added CT adjuvant to the immunization or not (Fig. 1d). As before, this contrasted with the finding in WT mice transferred with OTII CD4 T cells, where CT adjuvant was required (Fig. 1d). Next, we added NP-specific GFP ${ }^{+}$ $B$ cells from $\mathrm{B} 1-8^{\text {hi }}$ mice (C57BI/6 background) to the adoptive transfer protocol and immunized mice with NP-OVA with or without CT adjuvant. ${ }^{40}$ Using immunohistochemical analysis of frozen tissue sections, we found distinct GC reactions in the PP, resulting in the formation of antigen-specific IgA plasma cells $\left(\mathrm{GFP}^{+}\right)$in the gut LP (Fig. 1e). This was further confirmed by FACS analysis, where NP-specific B-cell expansion was observed only in PP, but not in MLN or spleen of orally immunized mice (Fig. 1e). We observed similar GC B-cell $\left(\mathrm{GL}^{+}\right)$and Tfh-cell $\left(\mathrm{CXCR} 5^{+} / \mathrm{PD}-1^{+}\right)$ frequencies as well as GFP ${ }^{+} \mathrm{NP}$-specific plasma blasts/plasma cells $\left(\mathrm{GFP}^{+} \mathrm{CD}_{138^{+}}\right)$in PPs from both unadjuvanted and CTadjuvanted mice (Fig. 1e). Thus, CT adjuvant was not required for a strong PP and gut LP IgA response in our adoptive transfer model. This experiment also demonstrated that TCR-Tg CD4 T cells transferred into either BALB/C- or $\mathrm{C} 57 \mathrm{BI} / 6 \mathrm{nu} / \mathrm{nu}$ mice establish a distinct and different regulatory environment not observed in the PPs of WT mice. That the anti-OVA gut IgA SFC response was truly CD4 T-cell dependent was confirmed by the lack of response in nu/nu mice without transfer of TCR Tg CD4 T cells, which also did not increase the level of total IgA SFC activity in the gut (Fig. 1f).

Two CD4 T-cell populations are required for gut LP IgA responses Previous publications have suggested an important role of Tregs for gut IgA responses, albeit conflicting findings have suggested that Th17 cells, rather than Tregs, are central to gut $\operatorname{lgA}$ responses. ${ }^{5,6,8}$ To address the need for Tregs in our model we sorted the splenic DO11.10 CD4 T-cell populations in $\mathrm{CD} 25^{+}$and CD25- CD4 T-cells prior to transfer into BALB/c nu/nu mice (Fig. 2a). Following oral immunization with OVA alone without adjuvant we found no $\lg A$ SFC response in the gut of the recipient mice and only when $\mathrm{CD} 25^{+}$and $\mathrm{CD} 25^{-} \mathrm{CD} 4 \mathrm{~T}$ cells were recombined at a 1:1 or even 1:9 ratio prior to transfer did we get a significant gut IgA response comparable to that seen with whole unsorted CD4 T cells (which have 1:9 ratio) (Fig. 2 b). Thus, it appeared that both populations were required to stimulate a gut LP IgA response. In sharp contrast, though, we observed a strong splenic IgG SFC and serum anti-OVA IgG response following oral immunizations in mice given $\mathrm{CD} 25^{-} \mathrm{CD} 4 \mathrm{~T}$ cells, indicating the presence of sufficient Tfh activity in the GALT to generate a specific lgG response (Fig. 2b). Phenotypic analysis of Tfh and Treg populations in the PPs revealed, indeed, a distinct presence of Th cells in CD25- CD4 Tcell transferred nu/nu mice, while a majority of $\mathrm{CD} 25^{+} \mathrm{CD} 4 \mathrm{~T}$ cells remained Foxp $3^{+}$Tregs even 26 days after transfer, demonstrating lineage stability (Fig. 2c). This lineage stability was also found with $\mathrm{KJ}_{126^{-}}$Treg cells, which, as expected, did not develop into Tfh cells following oral immunization (Fig. 2c). We concluded that two populations of CD4 T cells were required for induction of gut LP IgA responses, but only one was required for IgG anti-OVA responses following oral immunization with OVA. Importantly, because the nu/nu mouse lacks a thymus only antigen-induced pTregs can develop in the CD25 $5^{-}$CD4 T-cell transferred mice and, hence, the Tregs required for gut $\lg A$ responses following oral immunization were likely thymus-derived tTregs. This assumption was further supported by the high expression of Neuropilin-1 


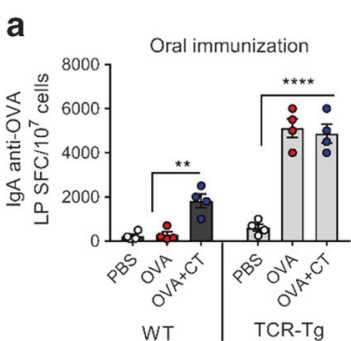

LP IgA anti-OVA SFC

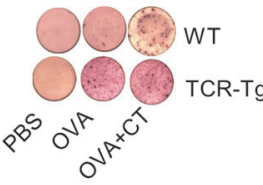

C



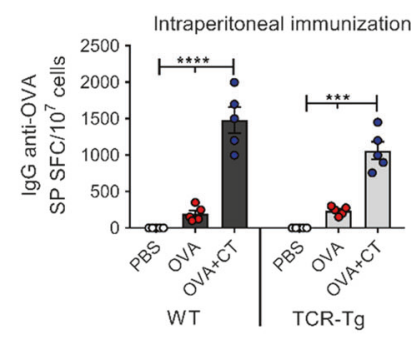

SP IgG anti-OVA SFC

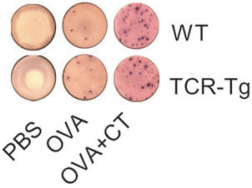

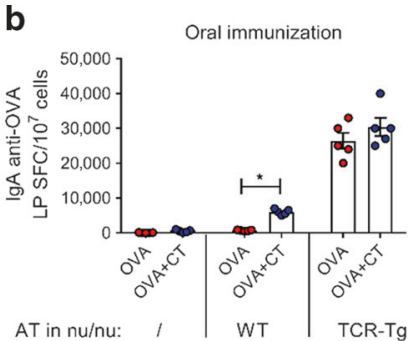

LP IgA anti-OVA SFC

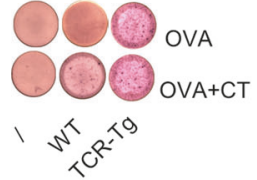

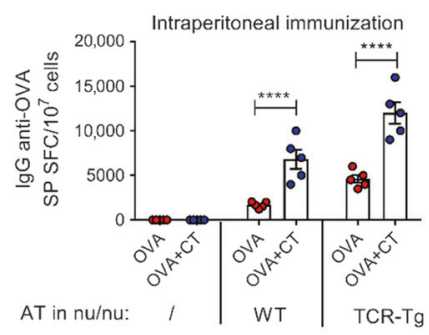

SP IgG anti-OVA SFC

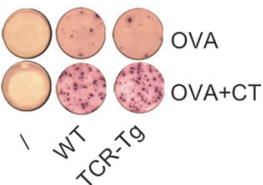

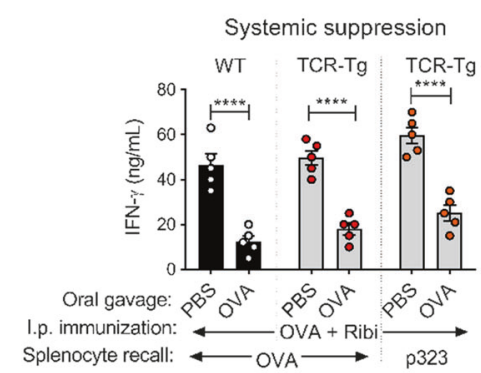


TCR-Tg $(\mathrm{C} 57 \mathrm{BL} / 6)$

AT in nu/nu
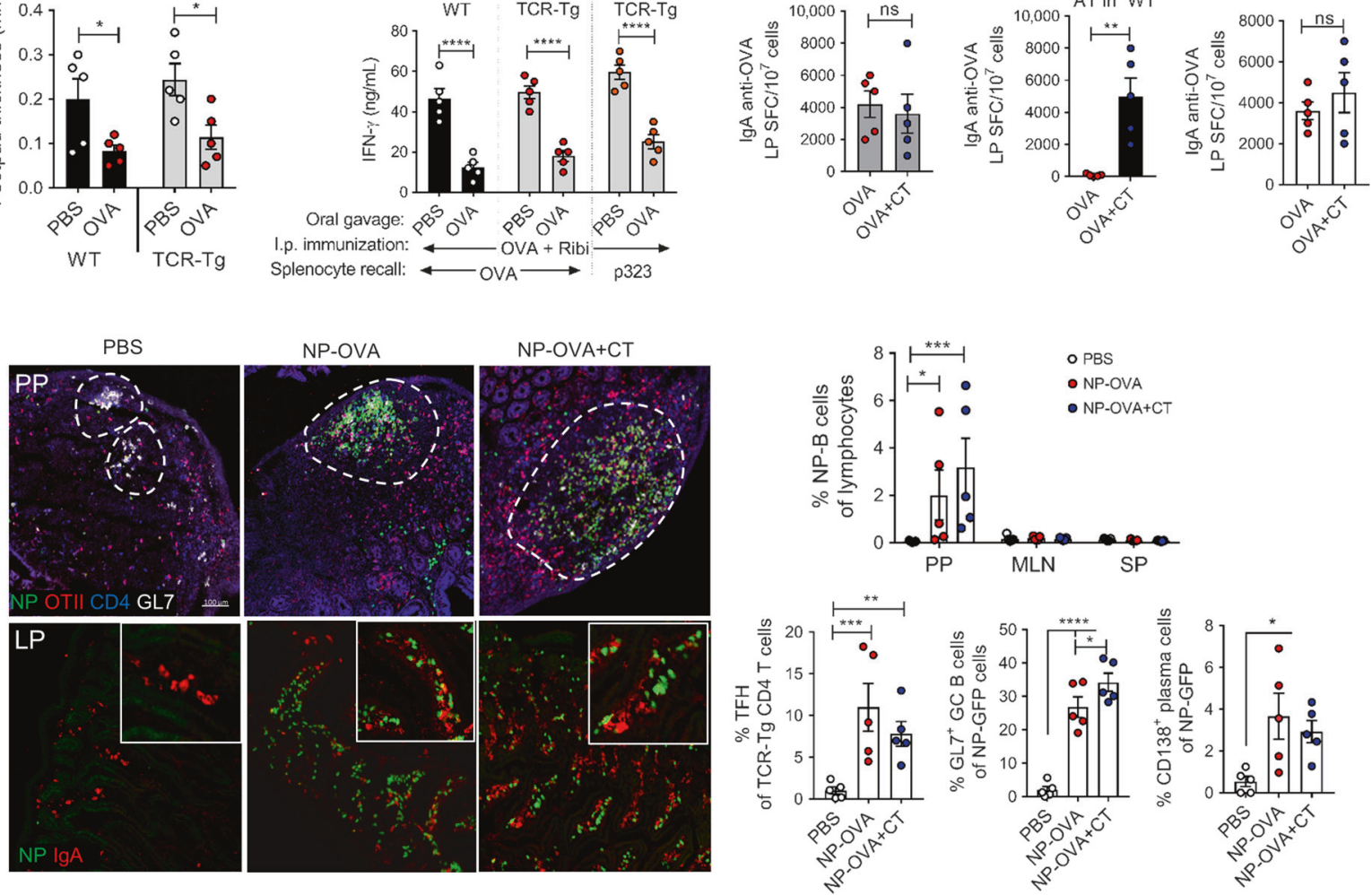

f
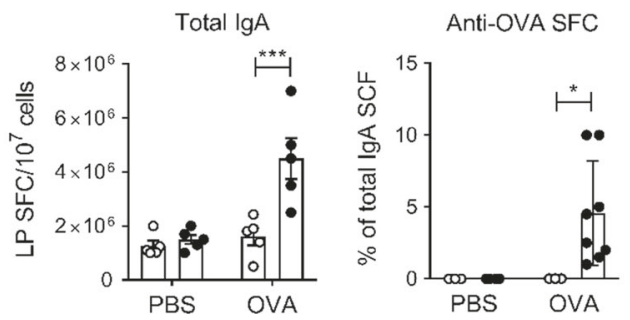

o nu/nu

- TCR-Tg AT nu/nu

(tTregs) (95\%) in the transferred $\mathrm{CD}^{2} 5^{+} \mathrm{GITR}^{+} \mathrm{Foxp}^{+} \mathrm{DO} 11.10$ CD4 T-cell population (Fig. 2a).

Gut microbiota is critical for Tfh function but appears dispensable for Tregs in PP

Next, we investigated to what extent the presence of the microbiota could have influenced the ability to develop gut $\lg \mathrm{A}$
LP responses because it is well known that germ-free mice produce little $\lg A .^{3,41}$ To this end, we eliminated intestinal bacteria with a cocktail of antibiotics (ABX) and compared anti-OVA LP IgA SFC responses with those observed in untreated DO11.10 mice upon oral OVA immunization. We found that OVA-specific LP IgA SFC responses were dramatically reduced $(75 \%)$ in $A B X$-treated mice and OVA-specific $\mathrm{KJ} 1-26^{+}$CD4 T cells were significantly 
Fig. 1 A model for gut IgA responses following oral immunization without the need for an adjuvant. Mice were immunized 3 times orally with $10 \mathrm{mg}$ of OVA w/wo $10 \mu \mathrm{g}$ of CT adjuvant or twice intraperitonally (i.p) with $50 \mu \mathrm{g}$ of OVA w/wo $2 \mu \mathrm{g}$ of CT and 10 days between immunizations. Lamina propria (LP) OVA-specific IgA spot-forming cell (SFC) and splenic (SP) IgG SFC responses were determined by ELISPOT 6-8 days after the final immunization. Control mice were given PBS (a-d, $\mathbf{f})$. IgA and IgG anti-OVA SFC values were analyzed in duplicates and are given as means \pm SEM $/ 10^{7}$ mononuclear cells of 5 mice in each group. a WT or DO11.10 TCR-Tg mice were orally or i.p immunized, $\mathbf{b}$ Nu/nu $\mathrm{BALB} / \mathrm{c}$ mice were adoptively transferred (AT) with highly enriched splenic CD4 T cells from WT or DO11.10 TCR-Tg mice and then orally or i.p immunized. c Oral tolerance was assessed as suppression of delayed-type hypersensitivity (DTH) in the footpad or IFN $\gamma$-responses by splenic CD4 T cells to recall antigen (OVA or p323 peptide) in vitro following oral immunization with OVA alone. All mice received a systemic i.p sensitization with OVA in Ribi-adjuvant prior to analyzing DTH $(48 \mathrm{~h})$ and IFN $\gamma$-responses. Values are given as means mm \pm SEM (footpad thickness) or means $\mathrm{ng} / \mathrm{mL} \pm \mathrm{SEM}$ (IFN $\gamma$ ) in supernatants with 5 mice in each group. d Oral immunization of OTII TCR-Tg mice (left panel), or WT (middle panel) and nu/nu (right panel) C57BL/6 mice after AT of highly enriched splenic TCR-Tg CD4 T cells. e Nu/nu C57BL/6 mice were AT with highly enriched splenic OTII TCR-Tg CD4 T cells together with NP-GFP ${ }^{+}$B cells from B1-8 ${ }^{\text {hi }}$ mice (C57BL/6). Mice were unimmunized (PBS) or orally immunized twice with NP-OVA w/wo CT adjuvant. On day 15 sections of PP (upper left panel) and gut small intestinal LP (lower left panel) were analyzed by microscopy using frozen tissue sections and specific labeled antibodies, as indicated: GFP (green), CD45.1 ${ }^{+}$OTII (red), GL7 (white), CD4 (blue), and IgA (red). Germinal centers with NP-specific B cells (green) are indicated by a dotted line. The different tissues were also analyzed for responding GFP ${ }^{+} \mathrm{B}$ cells by FACS (upper right panel). Only PPs had expanding GFP ${ }^{+}$B cells while mesenteric lymph nodes (MLN) and spleen (SP) did not host responding B cells. The distribution of NP-specific B cells (GFP ${ }^{+}$) and OTII TCR-Tg Tfh (CD45.1) cells was determined by FACS using labeling for CD138 or GL7 and CXCR5, respectively (lower right panels). f Total IgA production and percentage of specific response in LP of nude mice or nude mice adoptively transferred with 1 million TCR-Tg CD4 T cells after peroral OVA or PBS treatment as in $\mathbf{b}$. These are representative data from one of three independent experiments giving similar results. Statistical significance was calculated using two way ANOVA with Tukey's multiple comparison test. ${ }^{*} p<0.05,{ }^{* *} p<0.01,{ }^{* * *} p<0.001,{ }^{* * *} p<0.0001$, ns $=$ not significant

reduced in the PPs compared with that found in untreated control DO11.10 mice (Fig. 3a). A detailed FACS analysis of the regulatory environment in the PPs of ABX-treated mice revealed that while both Tregs and Tfr frequencies were increased 3-4-fold, the Tfh population in PPs was decreased by almost threefold compared with untreated WT mice (Fig. 3a). However, in absolute numbers the reduction in Th cells was remarkable, while Tregs did not significantly change in numbers (Fig. 3a). Thus, ABX treatment dramatically influenced the ability to raise Tfh cells. A similar observation was made in germ-free mice, which also exhibited distinctly lower Tfh cell frequencies and increased Treg and Tfr levels in the PPs compared with that detected in conventionally raised mice (Fig. 3b). Noteworthy, it was the tTreg-, rather than the pTreg population, in PPs that appeared most independent of the microbiota, as the absolute number of tTregs was unaltered in GF mice, while pTregs were dramatically reduced (Fig. 3b). This indicated that the microbiota was crucial for the presence of the Tfh population in the PPs but that Tfrs and, in particular, tTregs appeared unaffected by the microbiota. Previous studies have indicated that only $\mathrm{KJ} 1-26^{+}$transgenic CD4 T cells that also expressed an endogenous TCR, i.e., dual-TCRs, could react to the microbiota and support a gut LP IgA SFC response to oral immunization with OVA. ${ }^{31,42,43}$ We confirmed this in our nu/nu model by sorting and selectively transferring either dual TCR, that supported an OVA IgA response, or single-TCR-specific $\mathrm{KJ}_{1}-26^{+}$or $\mathrm{KJ} 1-26^{-}$CD4 T cells, which both failed to support a response to oral OVA immunizations (Fig. 3c). Furthermore, transfer experiments with DO11.10 CD4 T cells on a SCID background, which cannot rearrange the TCRa-chain to express a dual TCR, further confirmed this conclusion by failing to respond with IgA as well as IgG responses to oral OVA immunizations (Fig. 3d). ${ }^{44}$ Thus, we concluded that a PP Tfh population was critically needed for GALT-induced $\lg A$ and $\lg G$ responses and that an adoptively transferred transgenic Tfh population appeared to recognize microbial antigens due to secondary rearrangements of their endogenous TCRa-chain. Of note, the sorting strategy using different antibodies (anti-CD4, anti-KJ1-26, or anti-CD25 Mabs) that bind to the surface of the CD4 T cells did not influence the performance of the transferred CD4 T-cell populations.

We have previously documented that a normal gut LP $\lg \mathrm{A}$ response requires repeated oral immunizations and that there is synchronization of clonally related B cells between multiple PPs. ${ }^{45}$ Because we observed that dual-TCR expression and recognition of the microbiota were required for an oral response to OVA we hypothesized that recognition of the microbiota helped to establish functional inductive sites with CD4 T cells in multiple PPs in nu/nu mice through expansion of T cells. To this end, we compared TCRTg CD4 T cells from D011.10 or DO11.10 scid for their ability to promote these inductive sites in the PPs in nu/nu mice. We found expansion of transferred dual-TCR DO11.10 cells even in the absence of an oral OVA immunization, whereas single-TCR DO11.10 ${ }^{\text {scid }}$ cells failed to do so (Fig. 3e). In fact, adding an oral immunization with OVA did not change the frequency or composition of $\mathrm{KJ} 126^{+} \mathrm{Tfh}$, Tfr, or Tregs in the PPs of recipient nu/nu mice (Fig. 3e). A detailed analysis of the frequencies of these subsets in proximal to distal PPs revealed that whereas Tfh and Tfr frequencies were increased distally, Treg levels were rather reduced (Fig. 3f). Oral OVA immunization, did not appear to affect Tfr levels, but increased Tfh and reduced Treg frequencies in proximal PPs to the same levels as those found in distal PPs (Fig. 3f). Hence, exposure to the microbiota expanded dual-TCR-Tg CD4 T cells in PPs, with distinct profiles depending on their position within the small intestine, and empowered these inductive sites with necessary OVA-specific effector and/or memory CD44 ${ }^{+}$Tfh cells that could rapidly expand in an antigen-specific manner in distal PPs following an oral immunization.

\section{Thymus-derived natural Tregs producing TGF $\beta$ are critically} needed for gut IgA CSR

Finally, we investigated the Treg requirement for IgA CSR in the PPs in some more detail. First, we sorted DO11.10 splenic Tregs into non-Tregs, tTregs, and pTregs and cultured these together with splenic B cells and antigen (p323 peptide) in vitro. When cocultured with splenic B cells in the presence of antigen ( $p 323$ peptide), we observed significant IgA production only in cultures hosting tTregs (Fig. 4a). Thus, the adoptively transferred splenic Tregs were predominantly tTregs and these were able to promote IgA production in vitro. Furthermore, sorted Tregs from PP were necessary and sufficient for IgA production when cocultured with PP B cells and OVA-peptide produced IgA (Fig. 4b). By contrast, IgG was produced in PP cocultures lacking tTregs, but not with Tregs alone (Fig. 4b). Moreover, the only PP T cells that expressed surface TGF $\beta 1$, as determined by flow cytometry, were Tregs, and subsequently IgA production correlated with TGF $\beta 1$-producing Tregs (Fig. 4b). By using the SMAD3-inhibitor SIS3 in the drinking water we confirmed that, indeed, TGF $\beta$ was the critical IgA-switch factor in vivo following oral immunizations. ${ }^{46}$ In fact, orally immunized SIS3-treated DO11.10 mice demonstrated a significant reduction $(60 \%)$ of the gut LP IgA response to OVA as compared to untreated mice (Fig. 4c). Thus, both in vitro and in vivo experiments argued for a critical role of tTregs producing TGF $\beta 1$ for gut IgA CSR. 
I Gribonika et al.
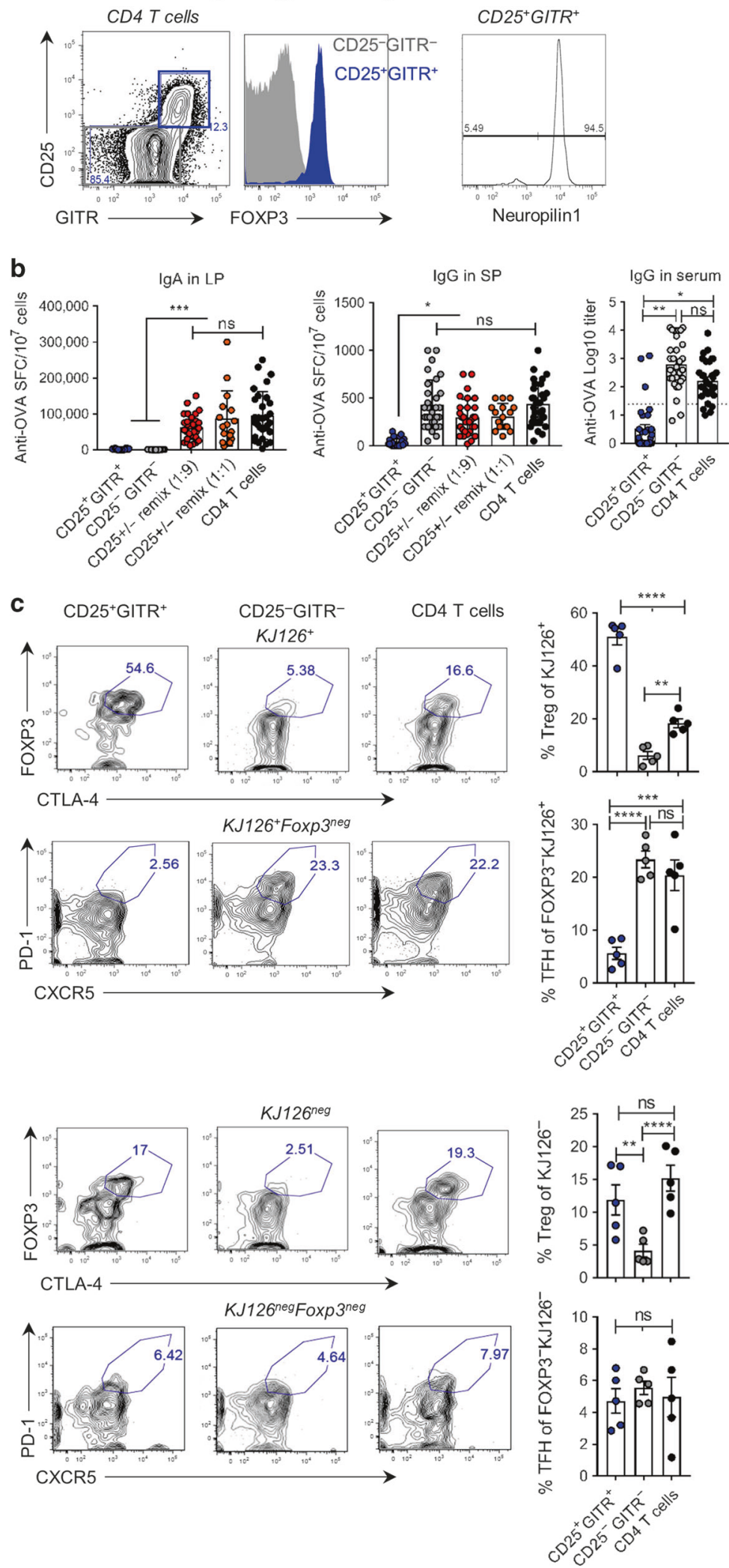

Frozen tissue sections were then analyzed by microscopy for the location of tTregs in the different compartments of the PP. We found that the frequency of Helios ${ }^{+}$Foxp $^{+}$tTregs was highest in the T-cell zone, but these cells were also present in the B-cell follicle, SED and GC (Fig. 4d).
To determine to what extent the tTregs needed to recognize antigen for lgA CSR in vivo we transferred splenic tTregs from WT or DO11.10 mice together with $\mathrm{CD}_{2} 5^{-} \mathrm{DO} 11.10 \mathrm{CD} 4 \mathrm{~T}$ cells into $\mathrm{BALB} / \mathrm{c}$ nu/nu mice and assessed the effect on specific serum IgG and gut LP IgA SFC responses to oral OVA immunization. We 
Fig. 2 Tfh and Tregs are both required in the PP for gut IgA responses. a Prior to adoptive transfer (AT) of splenic DO11.10 TCR-Tg CD4 T cells into nu/nu BALB/c mice we sorted them based on CD25 and GITR-expression. Whole TCR-Tg CD4 T cells or highly enriched CD25 $5^{+}$or CD25 TCR-Tg CD4 T cells were injected i.v separately or re-mixed together. b Mice were orally immunized with $10 \mathrm{mg}$ of OVA three times with 10 days apart and on day 26 the gut LP anti-OVA IgA or the splenic (SP) IgG SFC responses were determined by ELISPOT. SFC values were analysed in duplicates and are given as means $\pm \mathrm{SEM} / 10^{7}$ mononuclear cells of $16-30$ mice in each group. Serum anti-OVA IgG levels were determined by ELISA and given as mean $\log _{10}$-titers \pm SEM of 30 mice in each group. $c$ The distribution of CD4 T-cell subsets in PPs after AT of TCR-Tg CD4 T cells into nu/nu BALB/c mice followed by oral immunizations. The Tfh and Treg populations in the PPs were analysed by FACS using antibodies for CD4, KJ126 (OVA-specific), CXCR5, PD-1, Foxp3, and CTLA4. Values are given as mean \pm SEM frequencies of OVA-specific Tregs and Tfh TCR-Tg KJ126 ${ }^{+}$CD4 T cells and TCR-Tg KJ126 CD4 T cells. The data are from 1 representative experiment out of 5 independent experiments giving similar results $(\mathbf{a}, \mathbf{c})$ or pooled from three to five independent experiments (b). Statistical significance was calculated using two way ANOVA with Tukey's multiple comparison test. ${ }^{*} p<0.05,{ }^{* *} p<0.01,{ }^{* *} p<0.001,{ }^{* * *} p<0.0001$, ns $=$ not significant

observed that both WT and DO11.10 CD25 $5^{+}$tregs promoted a gut LP IgA SFC response, but unexpectedly WT tTregs were even more effective at supporting IgA CSR (Fig. 4e). Thus, tTregs did not need to recognize the immunogen to be effective at promoting IgA CSR in the PP. Concomitant with previous experiments we found that CD25- DO11.10 CD4 T cells were sufficient to support IgG responses in the absence of Tregs, as these cells hosted the Tfh population (Fig. 4e). We concluded that while Tfh cells had to recognize antigen the critical tTregs, responsible for IgA CSR, appeared to be independent of specific antigen-recognition, as WT CD4 T cells were even stronger supporters of IgA responses than OVA-specific TCR Tg CD4 T cells and given that the frequency of OVA-specific naïve T cells is very low in unimmunized mice. ${ }^{47}$ However, we did not investigate whether tTregs needed to recognize some other antigen, such as endogenous antigens in the PP.

\section{DISCUSSION}

The present study is the first to dissociate CD4 T-cell subsets required for gut IgA responses following oral immunization. By exploring an adoptive transfer model where TCR-Tg CD4 T cells were injected into nude mice we could sort and separate the CD4 T-cell subsets into distinct functions responsible for IgA CSR and Tfh activity, respectively. Rather unexpectedly this model system could be explored in the complete absence of a mucosal adjuvant (CT), only requiring administration of the antigen, and in this way we could take an unbiased approach to the regulation of gut $\lg A$ responses. After initially demonstrating that the $P P$ is the site for induction of the antigen-specific B-cell response following oral immunization we focused our study on this site. When sorted $\mathrm{CD} 25^{-}$CD4 T cells were transferred to nude mice we failed to detect a gut $\lg A$ response, whereas strong systemic $\lg G$ responses against OVA were observed. This implied that Tfh functions in PPs were present and functional, while IgA CSR was failing. By contrast when $\mathrm{CD} 25^{+} \mathrm{CD} 4 \mathrm{~T}$ cells were transferred to nude hosts Tfh cells were lacking and, thus, no $G C$ reaction occurred and no detectable IgA response was observed in the small intestinal LP. Narrowing down the options of $\mathrm{CD}_{2} 5^{+} \mathrm{T}$ cells to tTregs or pTregs, we identified that the former were critically needed and produced the IgA-switch factor TGF $\beta 1{ }^{11,13}$ This outcome is highly reminiscent of the observations made by Strober and co-workers in the early 1980 's identifying a division of labor between "switch T cells" and CD4 $T$ cells responsible for terminal differentiation of activated $B$ cells in the PP. ${ }^{4}$ Later, studies by Elson and co-workers demonstrated that Tregs were involved in gut IgA responses using CD4 T cells from the CBir1 TCR-Tg mouse, specific for flagellin from the microbiota. ${ }^{5}$ However, these investigators could not account for if the CD4 T-cell subset that controlled IgA CSR also was the Tfh cell subset that maintained the GC and terminally differentiated the activated B cells. Rather their studies supported the notion that Tregs provided all necessary helper functions for gut $\lg A$ responses and the concept of a Treg-lgA axis was proposed. ${ }^{5}$ However, these Tregs were CBir1-specific and had to be stimulated via TCR recognition of flagellin to promote $\lg \mathrm{A}$ production and, therefore, were antigen-restricted and not likely tTregs, but possibly pTregs stimulated in the gut. Here we instead provide compelling evidence that the TGF $\beta 1$-producing subset is, indeed, the tTreg, as $\mathrm{CD} 25^{+} \mathrm{CD} 4 \mathrm{~T}$ cells did not have to recognize OVA and were Neuropilin- ${ }^{+}$, Helios $^{+}$GITR $^{+}$Foxp3 $^{+}$CD4 T cells that effectively supported IgA CSR.

Also other investigators have used adoptive transfer models to get to grips with what is required for a gut LP $\lg A$ response. Fagarasan and colleagues explored transfers of Foxp $3^{+}$CD4 T cell into $\mathrm{CD} 3 \mathrm{e}^{-1-}$ mice, which gave rise to $\mathrm{GC}$ formations and $\operatorname{lgA}$ production that was linked to the appearance of Thh cells in PPs. ${ }^{8}$ This observation supported a Treg-lgA axis and advocated that there was conversion of Tregs into Tfh functions, supporting GC formations and IgA CSR. These authors proposed that converting cells were losing expression of Foxp3 and were generated from CD25 low Foxp3 $^{+}$with modest expression of mir-10a (microRNA, mir-10a, highly expressed by tTregs), and, therefore, likely pTregs that could undergo conversion to Tfh cells. ${ }^{17,48}$ Thus, plasticity in pTregs gave rise to $\lg \mathrm{A} C S R$ and subsequent Tfh functions. However, also Th17 cells were observed to have the ability to convert into Tfh cells in PPs and support gut IgA responses. ${ }^{6}$ CD4 $\mathrm{T}$ cells from IL-17 reporter mice (II17a $\left.a^{\mathrm{Cre}} \mathrm{R} 26 \mathrm{R}^{\mathrm{eYP}}\right)$ were transferred into $\mathrm{Trra}^{-1-}$ mice and found to convert into Tfh cells, downmodulating RoRyt and supporting $\lg A$ production in the PP. Experiments undertaken in chimeric Tcra $x$ roryc mice suggested that Th17 cells, rather than IL-17A, were required for gut $\lg \mathrm{A}$ responses to oral CT immunizations. ${ }^{6}$ Taken together these studies argued that Tfh populations in the PP could have a heterogenous origin, and that both Tregs and Th17 cells exhibited plasticity and could convert into Tfh cells. ${ }^{49,50}$ But, because of the nature of their model systems they were not in a position to separate IgA CSR from the Tfh functions.

A critical factor that enabled the current study and allowed the separation of IgA CSR and Tfh functions can be attributed to dual TCR-receptor recognition. ${ }^{51}$ It is well described that rearranging the a-chain in TCR-transgenic mice leads to $T$ cells with dual receptors, a phenomenon that apparently is not uncommon in also naturally occurring $T$ cells. ${ }^{52}$ Nevertheless, this way we could identify the OVA-specific CD4 T cells that also recognized antigens from the microbiota, which was further supported by experiments where antibiotic treatment resulted in less Tfh activity compared to untreated mice following oral immunization. Thus, whereas the tTreg contribution was restricted to IgA CSR the Tfh subset was essential for GC formation and terminal differentiation of $\lg A B$ cells in PP. Indeed, even without immunization, we found significant proliferation of transferred TCR-Tg CD4 T cells and all $\mathrm{PPs}$ along the entire small intestine of recipient nu/nu mice were populated with Tfh cells. We think this development was particularly important and helps explain why we could separate IgA CSR from Tfh requirements in our model. Along these lines, we, therefore, consider this as an act of "normalization" of the inductive conditions in the recipient mouse gut with access to CD40L-expressing Tfh cells in multiple PPs. This notion is based on our earlier studies of synchronization of gut $\lg A$ responses requiring distribution of $B$ cells with the same clonal origin to 

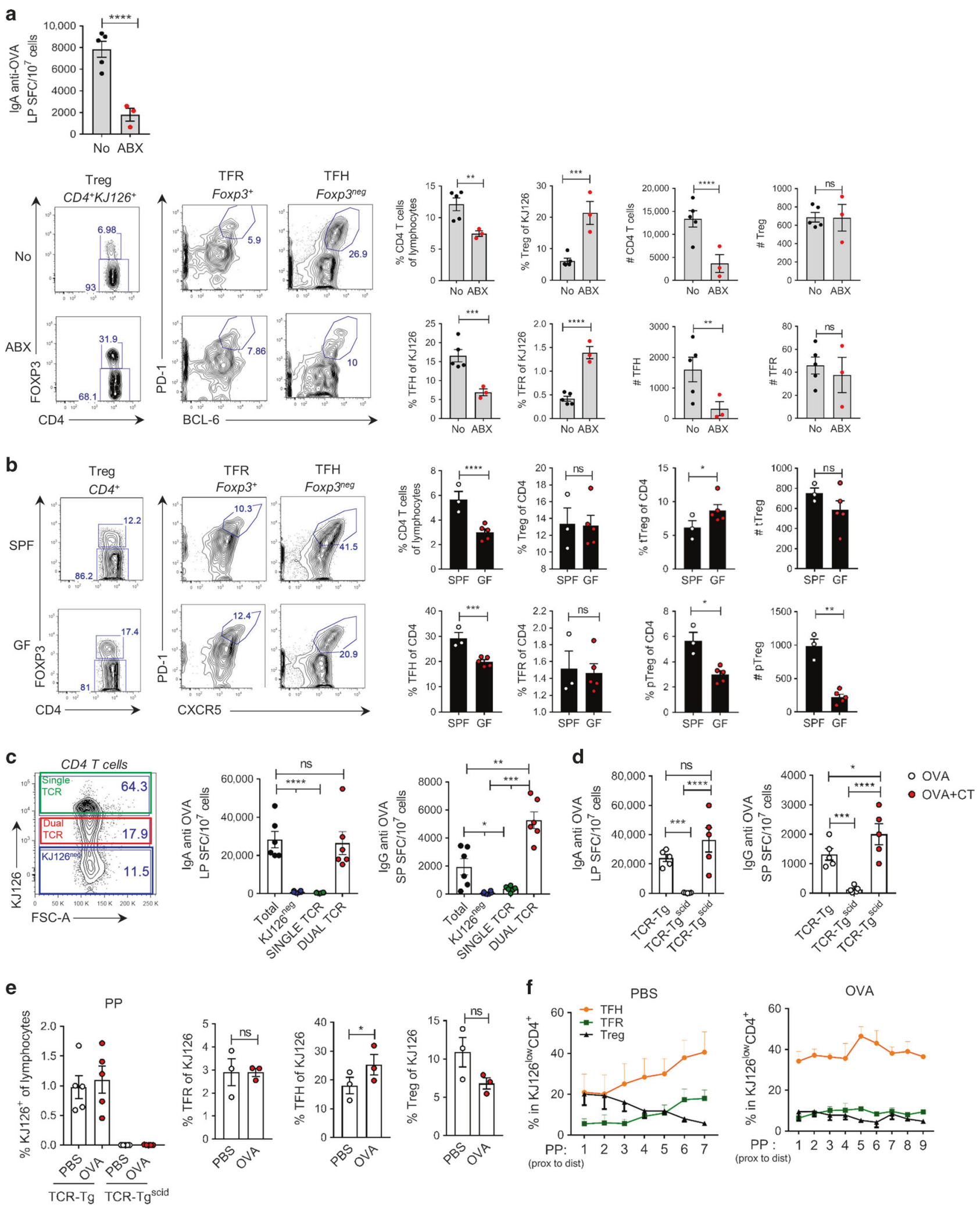

f
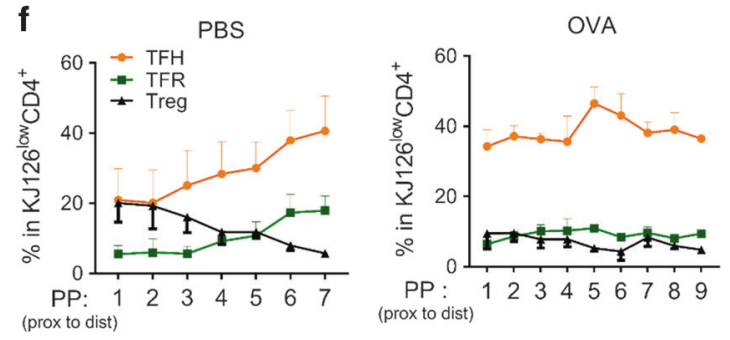

multiple PPs following oral immunization. ${ }^{45}$ This led us to hypothesize that activated antigen-specific B-cell clones in multiple PPs need to expand by reutilizing existing GC with Tfh cells expressing CD40L, possibly in a non-cognate fashion. By contrast, oral immunization with CD4 T cells taken from DO11.10 TCR-Tg mice on a scid background failed to generate a widespread distribution of Tfh cells to multiple PPs. Hence, the reason why D011.10 TCR-Tg scid CD4 T cells did not work is the failure to "normalize" the PPs with CD44 ${ }^{+}$antigen experienced Tfh cells. Oral immunizations were simply ineffective in generating the required Tfh cells in multiple PPs, unless $C T$ adjuvant was used, which normally promote the presence of $\mathrm{CD} 44^{+}$Tfh cells in 
Fig. 3 The presence of Tfh cells in the PP is secured through dual TCR expression and recognition of the microbiota. a To determine to what extent the microbiota influenced the response of the OVA-specific TCR-Tg CD4 T cells following oral immunizations of DO11.10 TCR-Tg mice we employed a mix of antibiotics (ABX) given in the drinking water for 2 weeks prior to and during the whole experiment. a, c-f The animals were orally immunized with $10 \mathrm{mg}$ of OVA three times with 10 days apart and on day 26 the gut LP anti-OVA IgA SFC response was determined by ELISPOT. a SFC values were analysed in duplicates and are given as means $\pm \mathrm{SEM} / 10^{7}$ mononuclear cells of 3-5 mice in each group. a FACS analysis to determine the distribution of $\mathrm{CD}^{+} \mathrm{KJ}^{2} 26^{+}$OVA-specific T-cell subsets in the PPs using labeled antibodies specific for CD4, KJ126 (OVA-specific), CXCR5, PD-1, Bcl-6, Foxp3 and CTLA4. The frequencies and the absolute number within PP of Tregs, Tfh and Tfr were assessed by gating, as described, and given as mean \pm SEM of CD4 T cells, or as indicated, of 3-5 mice in each group. $\mathbf{b}$ A corresponding FACS analysis of the CD4 T-cell subsets in PPs in unimmunized germ-free (GF) and conventionally raised specific pathogen-free (SPF) mice. The frequencies of Tregs, Tfh and Tfr and absolute numbers of Tregs were assessed by gating, as described, and given as mean \pm SEM of CD4 T cells, or as indicated, of 5 mice in each group and using the specific antibodies as in a. The relative presence of pTregs or tTregs in the PPs was assessed using a labeled Neuropilin-1 antibody. $\mathbf{c}$, $\mathbf{d}$ We tested the requirement for dual-TCR expression and recognition of the microbiota in the DO11.10 TCR-Tg CD4 T-cell subsets that were adoptively transferred (AT) to nu/nu BALB/c mice. (c) Prior to AT we sorted the splenic CD4 T cells into three categories based on the expression of the OVA-TCR. Nu/nu mice received whole CD4 T cells, $\mathrm{KJ}^{126^{-}}$, single-TCR ${ }^{+}$or dual TCR ${ }^{+}$ expressing CD4 T cells from DO11.10 TCR-Tg mice or (d) whole CD4 T cells from DO11.10 TCR-Tg on a SCID background (DO11.10 TCR-Tgsid) followed by three oral immunizations with OVA (c, d) or OVA plus CT adjuvant (d), as indicated. Next we determined the OVA-specific lamina propria (LP) IgA SFC or splenic (SP) IgG SFC responses by ELISPOT. Values are given as means \pm SEM $/ 10^{7}$ mononuclear cells of 5 mice in each group c, d. e Expansion and distribution of TCR-Tg CD4 T cells in recipient nu/nu BALB/c mice after AT of CD4 T cells from DO11.10 or DO11.10 scid mice followed by oral immunization with OVA as in a. Cell subsets in the PP were defined as Tregs, Tfr or Tfh cells as in a and assessed by FACS and their frequencies are given as $\% \pm$ SEM of KJ126 ${ }^{+}$CD4 T cells. $f$ Expansion and distribution after AT of TCR-Tg CD4 T cells in individual PPs in nu/nu BALB/c mice without immunization (PBS) or 5 days following a single oral OVA immunization. Frequencies and location to individual PPs from proximal to distal small intestine is given for Tfh, Tfr and Tfh cells as defined in a. The data are representative of three independent experiments giving similar results. Statistical significance was calculated using two way ANOVA with Tukey's multiple comparison test. ${ }^{*} p<0.05,{ }^{* *} p<0.01,{ }^{* * *} p<0.001,{ }^{* * * *} p<0.0001$, ns $=$ not significant

multiple PPs. By contrast, in OVA-specific TCR-Tg CD4 T cells, on a $\mathrm{BALB} / \mathrm{C}$ or a $\mathrm{C} 57 \mathrm{BI} / 6$ background, Tfh cells were generated through dual-TCR recognition of microbial antigens and in this way the microbiota acted as a "natural adjuvant" and promoted OVA-specific Tfh cells in multiple PPs.

In the present study we did not identify where the IgA CSR occurred in the PP, but we have previously shown that T-cellindependent IgA CSR can occur at a pre-GC stage as observed in PPs of CD40-deficient mice lacking GCs completely. ${ }^{53}$ Here we have studied a T-cell dependent response. Whereas it has been well documented that T-cell dependent (TD) B-cell responses undergo CSR in the GC in systemic peripheral lymph nodes, this option also has been favored for IgA CSR in the PP, but recent studies have instead demonstrated that the SED could be a site for pre-GC IgA CSR. ${ }^{23}$ We found the presence of tTreg (Foxp3 ${ }^{+}$ Neuropilin- ${ }^{+}$Helios $^{+} \mathrm{CD}^{2} 5^{+} \mathrm{GITR}^{+}$CD4 T cells) in all compartments of the PP, including the GC and SED, but most tTregs were found in the T-cell zone. It is conceivable that activated $B$ cells are exposed to tTregs and TGF $\beta$ when located to the T-Bcell border prior to manifest GC formation or, more likely, in the GC or even in the SED. However, to what extent the SED is required for IgA CSR following recognition of a TD-antigen is still poorly known. ${ }^{3}$ We recently identified that during an ongoing Bcell response to oral immunization significant numbers of specific $B$ cells were present in the SED region and these were $\mathrm{CCR6}^{+}{ }^{2}$ In fact, these $\mathrm{B}$ cells could rapidly bind luminal antigen and migrate toward the GC via the SED region, suggesting an important M-cell-B-cell antigen transporting pathway in the PP, which could also involve IgA CSR. Nevertheless, additional studies are needed to better dissect these options. Indeed, our current study confirms the findings of Strober and co-workers that identified a "switch T cell" to be responsible for IgA CSR, eliminating the further quest for the cellular source of TGF $\beta$, as we demonstrate the central role of tTregs for IgA CSR. ${ }^{4}$ Similar to the results of other researchers we observed a strong correlation between Tregs and TGF $\beta$, and, indeed, tTregs produced TGF $\beta 1$. However, tTregs could also provide av $\beta 8$ integrin, which can activate latent TGF $\beta$ produced by other cells and in this way direct IgA CSR. ${ }^{24}$ To more precisely investigate the site for $\lg A$ CSR in the PP it could be advantageous to use TGF $\beta$ RII-signaling via SMAD2/3-activation in the $B$ cells. ${ }^{54,55}$ Possibly a model system that explores phosphorylation of $\mathrm{SMAD2/3}$ or downstream events restricted to $B$ cells could be used. ${ }^{56,57}$
We could clearly separate tTreg from pTreg functions and the tTregs were the TGF $\beta 1$-producers and responsible for the IgA CSR function in the PPs. Although much debated the consensus today is that tTregs, but not pTregs, are fairly stable. ${ }^{58}$ Our results confirm this notion as the transferred tTregs maintained lineage stability in the nu/nu hosts and did not switch to Th functions because no GC or antibody responses were recorded following oral immunizations of mice injected with $\mathrm{CD} 25^{+} \mathrm{CD} 4 \mathrm{~T}$ cells. Furthermore, these tTregs appeared not to depend on the microbiota as their absolute numbers were not changed in antibiotic treated mice and they were also present in germ-free mice. Noteworthy, the recently described Tfr population, which is thought to arise from tTregs, could be involved in the IgA CSR, albeit these cells are better known for production of IL-10. ${ }^{59,60}$ As we monitored Tfr development in PPs following oral immunization, as well as in response to antibiotic treatment, it appeared that these cells were unresponsive to both interventions, similar to the tTreg population. A recent study reported that human Tfrs are located to the T-B border where they express a latent form of TGF $\beta$, which could influence IgA CSR from outside of the GC. ${ }^{61}$ Nevertheless, future studies are needed to identify if Tfrs or tTregs are controlling IgA CSR and exactly how and where this event takes place in the PP.

\section{MATERIALS AND METHODS}

Mice

Breeding pairs of OVA TCR-transgenic (DO11.10) mice on a $\mathrm{BALB} / \mathrm{C}$ or CB17.scid background, that recognizes the 323-339 peptide fragment of OVA in the context of I-Ad, were kindly provided by Dennis Y. Loh (Howard Hughes Medical Institute, Washington University, St Louis, MO) and bred and housed under pathogen-free conditions at the Department of Experimental Biomedicine, University of Gothenburg, Sweden. Syngeneic BALB/c mice were obtained from Taconic (Denmark) and used as wild-type (WT) control mice. BALB/c nude mice were obtained from Charles River Laboratories (Germany) and C57BL/ 6 nude mice were purchased from Taconic (USA). OTII-ly5.1, B1$8^{\text {hi }}, 40$ and C57BL/6 mice were bred and housed under pathogenfree conditions at the Department of Experimental Biomedicine, University of Gothenburg, Sweden, C57BL/6 germ-free mice were kindly provided by Fredrik Bäckhed (University of 



d


e

Isolated splenic CD4 T cell fractions


Gothenburg). Age- and sex-matched mice were routinely used with mice ranging from 6 to 12 weeks of age at the onset of the experiments. Mice were either littermates and/or cohoused for at least 2 weeks prior the experiment.
Immunizations

To induce local IgA immunity mice were orally immunized with 10 mg of OVA protein grade $V$ (Sigma) dissolved in $0.5 \mathrm{~mL}$ of PBS and containing $3 \%(\mathrm{w} / \mathrm{v}) \mathrm{NaHCO}_{3}$ with or without the addition of $10 \mu \mathrm{g}$ 
Fig. 4 IgA CSR in the PP is mediated by TGF $\beta$ and requires tTregs. A detailed analysis of the Treg requirement for IgA CSR was undertaken. a DO11.10 TCR-Tg CD4 T cells were sorted from the spleens of DO11.10 mice based on CD25, GITR, and Neuropilin-1 expression into the tTregs, pTregs or non-Treg CD4 T subsets and the individual pooled cells or naïve splenic CD4 T cells were co-cultured with naïve splenic B cells (lgD ${ }^{+}$) together with OVA peptide p323 for 8 days. Total IgA in culture supernatants was assessed by ELISA and values are given as lgA ng/mL \pm SEM of triplicate cultures. b A similar experiment was set up with sorted and pooled CD4 T-cell subsets from the PP of DO11.10 mice. Highly enriched PP whole CD4 T cells, non-Tregs or tTregs were co-cultured with naïve PP B cells (lgD ${ }^{+}$) and OVA peptide p323 for 6 days. Total IgA or lgG1 in culture supernatants were assessed by ELISA and values are given in $\mathrm{ng} / \mathrm{mL} \pm$ SEM of triplicate cultures as in a. FACS analysis of the Treg surface expression of TGF $\beta 1$ as determined in the cultures described above with CD4 T cells and B cells. The analysis is presented as a histogram. c The TGF $\beta$-blocking- effect of the inhibitor SIS3 in the drinking water on gut IgA responses in orally OVA immunized DO11.10 TCRTg mice was determined. OVA-specific gut LP IgA SFC responses are given as means \pm SEM $/ 10^{7}$ mononuclear cells of 5 mice assessed on day 26 following 3 oral immunizations. d Frozen tissue sections of PP were prepared from C57BL/6 mice and labeled with antibodies specific for PP compartmentalization: CD11c (magenta), GL-7 (cyan), CD4 (green), CCR6 (red) and B220 (blue); for Treg detection: Foxp3 (red), CD4 (green) and Helios (white). Helios ${ }^{+}$Foxp ${ }^{+} \mathrm{CD}^{+}{ }^{+} \mathrm{T}$ cells (marked by blue arrowheads) were counted per visual section and found to locate to the subepithelial dome (SED), germinal center (GC), B-cell follicle (B) and T-cell zone (T). e Treg-negative CD25 $5^{-}$Foxp3 $3^{-}$from DO11.10 TCR-Tg mice were sorted and adoptively transferred alone or together with CD25 $5^{+}$Foxp $3^{+}$tTregs from WT or DO11.10 TCR-Tg mice. Following oral immunizations with OVA serum anti-OVA IgG antibodies or gut LP anti-OVA IgA SFC responses were assessed on day 30 . These data are from one representative experiment out of $4(\mathbf{a}-\mathbf{d})$ or $2(\mathbf{e})$ independent experiments giving similar results. Statistical significance was calculated using two way ANOVA with Tukey's multiple comparison test. ${ }^{*} p<0.05,{ }^{* *} p<0.01$, ${ }^{* * *} p<0.001$, ${ }^{* * * *} p<0.0001$, ns $=$ not significant

cholera toxin (CT) adjuvant (List Biological Laboratories, Campell, CA) to each dose. The mice were given three oral administrations, 10 days apart, using intragastric intubation. Parenteral immunizations in $0.5 \mathrm{~mL}$ PBS were done i.p twice with $50 \mu \mathrm{g}$ OVA w/wo 2 $\mu \mathrm{g} C \mathrm{~T}$.

CD4 T cell, Treg or B-cell isolation and adoptive transfer to nude mice

Single cell suspensions of spleen and MLN were depleted of erythrocytes by hypotonic lysis. Cells were enriched for CD4 ${ }^{+}$ $\mathrm{T}$ cells by negative selection using StemCell EasySep ${ }^{\mathrm{TM}}$ Mouse $\mathrm{CD}^{+} \mathrm{T}$ Cell Isolation Kit (cat.\# 19852). The purity of $\mathrm{CD}^{+}$cells was typically $95-99 \%$. Treg isolation was undertaken using StemCell EasySep $^{\mathrm{TM}}$ Mouse CD25 Regulatory T Cell Positive Selection Kit, for positive selection (cat.\# 18782), with a cell purity typically $90-95 \%$ or cells were sorted based on surface expression of CD25 and GITR. StemCell EasySep ${ }^{\mathrm{TM}}$ Mouse B Cell Isolation Kit, for negative selection (cat.\# 19854) supplemented with $2 \mu \mathrm{g}$ anti-mouse Kchain biotinylated antibody (187.1) from BD Biosciences was used for B-cell enrichment and a purity of typically $95 \%$ was achieved. Cells in PBS were injected i.v. $\left(200 \mu \mathrm{L} / 2 \times 10^{6}\right.$ /mouse $) 1$ day before the first immunization.

\section{Assessment of oral tolerance}

Oral tolerance was assessed at 7 days after the last feeding by priming i.p. with $200 \mu \mathrm{g}$ of OVA in Ribi adjuvant (TriChem Aps, Copenhagen, Denmark) followed by an i.p. boost 14 days later with $200 \mu \mathrm{g}$ of OVA given alone. Control mice were fed PBS only. Delayed-type hypersensitivity (DTH) reactions were assessed and the degree of suppression induced by a single oral feeding of $\mathrm{Ag}$ was determined after an i.p. sensitization with OVA in Ribi adjuvant followed by a s.c. challenge into the rear footpad with $100 \mu \mathrm{g}$ heat aggregated OVA. The increase in footpad thickness was measured $48 \mathrm{~h}$ later.

\section{Antibiotic treatment}

DO11.10 OVA TCR-Tg mice were kept on antibiotics (vancomycin $0.5 \mathrm{~g} / \mathrm{L}$, neomycin $1 \mathrm{~g} / \mathrm{L}$, ampicillin $1 \mathrm{~g} / \mathrm{L}$, metronidazole $1 \mathrm{~g} / \mathrm{L}$, sucrose $20 \mathrm{mg} / \mathrm{L}$ ) in drinking water for 2 months (including duration of the experiment). Water was changed every 3rd day.

\section{TGF $\beta$ inhibition in vivo}

The experimental group of D011.10 OVA TCR-Tg mice was given $20 \mathrm{mg} / \mathrm{L}$ SIS3 (SMAD3 inhibitor, Tocris Bioscience) in the drinking water and the water with inhibitor was changed every 3rd day.

\section{Preparation of LP lymphocytes}

The isolation procedure to obtain small intestinal LP lymphocytes has been described in detail. ${ }^{62}$ Briefly, after removing the PP, the small intestines were cut into small pieces and washed in $\mathrm{Ca}^{2+}$ and $\mathrm{Mg}^{2+}$-free Hanks' balanced salt solution (CMF-HBSS, Gibco). Thereafter, the tissue pieces were incubated in CMF-HBSS containing $5 \mathrm{mM}$ EDTA (Merck, Darmstadt, Germany) to remove epithelial cells and intra-epithelial lymphocytes and finally the LP lymphocytes were extracted by digesting the tissue by repeated incubations with $100 \mathrm{U} / \mathrm{mL}$ of collagenase-D (Roche) in RPMI 1640 (Flow Laboratories, Irvine, Scotland).

\section{Serum antibody titers}

Mice were bled at sacrifice and serum samples were stored at -80 ${ }^{\circ} \mathrm{C}$ and analyzed individually in flat-bottom 96-well MaxiSorp microtiter plates (Nunc, Roskilde, Denmark). Wells were coated with $200 \mu \mathrm{g} / \mathrm{mL}$ of OVA (Sigma) in PBS at $4{ }^{\circ} \mathrm{C}$ overnight. After washing the plates in PBS and blocking with $0.1 \% \mathrm{BSA} / \mathrm{PBS}$, sera were serially diluted in $0.1 \% \mathrm{BSA} / \mathrm{PBS}$ and aliquots were added to corresponding sub-wells. The plates were kept at $4{ }^{\circ} \mathrm{C}$ overnight and following washing in PBS the plates were incubated with APconjugated isotype-specific goat anti-mouse antibodies (Southern Biotechnology, Birmingham, AL). Plates were washed and the phosphatase substrate, p-nitrophenyl phosphatase (NPP) (Sigma) was added to each well. The reaction was read at $405 \mathrm{~nm}$ using a Titertek spectrophotometer (Flow Laboratories, Irvine, Scotland). The antibody titers were defined as the interpolated dilutions of the samples giving rise to an absorbance on the linear part of the curve of 0.4 (serum) above background.

\section{ELISPOT assay}

The detection of Ag-specific LP or SP SFC activity was performed as described. ${ }^{63}$ Briefly, 96-well plates (Multiscreen HTS, Millipore) were coated with OVA (Sigma) at $200 \mu \mathrm{g} / \mathrm{mL}$ or purified $\operatorname{lgA}(\mathrm{BD})$ at $5 \mu \mathrm{g} / \mathrm{mL}$ in PBS. After blocking with $0.1 \% B S A / P B S$, duplicate wells were incubated with mononuclear cells from the LP or spleen. The cells were incubated for $3 \mathrm{~h}$ at $37^{\circ} \mathrm{C}$ and $5 \% \mathrm{CO}_{2}$. After thorough washing in PBS/0.05\% Tween 20 specific antibodies in $100 \mu \mathrm{L} /$ well were added sequentially to the wells starting with; AP-conjugated goat anti-mouse IgG (Southern Biotech) for spleen and APconjugated goat anti-mouse IgA (Southern Biotech) for LP SFCs. The bound antibodies marking single antibody-producing cells were visualized by adding $50 \mu \mathrm{L} /$ well of Sigmafast ${ }^{\mathrm{TM}}$ BCIPR/NBT B5655 1 tablet/10 mL deionized water (Sigma). SFCs were counted using an Immunospot Analyzer (CTL).

\section{Flow cytometric analysis}

For analysis of intracellular or membrane bound molecules we used freshly isolated cells that were labeled with specific fluorochrome-conjugated antibodies. All samples were preincubated with 2.4G2 (anti-Fc-receptor) to avoid unspecific labeling and the following antibodies were used; anti-CD4- 
BV785 (RMA4-5), anti-KJ126-PE (KJ1-26), anti-Va2-PE (B20.1), antiPD1-PECF594 (EH12.1), anti-GITR-PECy7 (DTA-1), anti-BCL6-PE (K112-91), anti-CD19-PECF594 (1D3), anti-CD25-PerCPCy5.5 (PC61), anti-CD138-PE (281-2) obtained from BD Biosciences, anti-FOXP3-AF647 (150D), anti-Neuropilin-1-APC (3E12), antiNeuropilin-1-BV421 (3E12), anti-PD1-BV711 (29F.1A12), antiCD44-BV570 (IM7), anti-CTLA4-BV421 (UC10-4B9), anti-HeliosPerCPCy5.5 (22F6), anti-TCRb-APC-Cy7 (H57-597) obtained from Biolegend, anti-MHCII (I-A/I-E)-AF700 (M5/114.15.2), obtained from eBioscience and purified clonotypic KJ1-26 antibodies conjugated with AF488. To exclude dead cells 7-Aminoactinomycin D (Sigma Aldrich) or Live/Dead Aqua (molecular probes, Life Technologies, Thermo Scientific) positive cells were excluded from the analysis. Intracellular labeling was performed using FOXP3/transcription factor staining kit (eBioscience) according to the manufacturer's instructions. Events were collected and analyzed by using an LSR-II or LSR Fortessa X-20 (BD Biosciences) and samples were analyzed by FlowJo software (Tree Star). Cultured Tregs were labeled with PE-conjugated anti-mouse LAP/TGF $\beta-1$ (TW7-16B4) from BD.

\section{Cell sorting}

Single cell suspensions enriched for CD4 T cells using StemCell EasySep $^{\text {TM }}$ Mouse CD4 ${ }^{+}$T Cell Isolation Kit (cat.\# 19852) and stained for surface markers anti-CD44-AF700 (IM7), anti-CD4BV785 (RM4-5), anti-KJ126-PE (KJ1-26), anti-GITR-Pe-Cy7 (DTA-1), anti-CD25-PerCP-Cy5.5 (PC61) obtained from BD and antiNeuropilin-1-BV421 (3E12) obtained from Biolegend. Single cell suspension enriched for B cells using StemCell EasySep Mouse Bcell isolation kit (\#cat. 19854) was stained for anti-CD19-PeCy7 (1D3) from BD and anti-lgD-PerCP-Cy5.5 (11-26c.2a) from Biolegend. Flow cytometric cell sorting was done using a FACSAria III (BD Biosciences). Cells were sorted into tubes that had been precoated with $0.1 \%$ BSA/PBS overnight.

In vitro cell cultures

Sorted or enriched D011.10 CD4 T-cell subsets (pTregs, tTregs, Treg $^{\text {neg) }}$ and naïve B cells from PP or spleen were cultured in various combinations under stimulation of $1 \mu \mathrm{M}$ p323. Cells were plated in triplicate cultures in round-bottom 96-well plates (Nunc A/S) with 50,000 B cells and 30,000 T cells in $200 \mu \mathrm{L}$ complete Iscove's medium. Cultures hosted in addition 5000 accessory cells per well. After $7-8$ days at $37^{\circ} \mathrm{C}$ in $5 \% \mathrm{CO}_{2}$, supernatants were harvested for detection of total $\lg A$ or $\lg G 1$ using ELISA. Briefly, flat-bottom 96-well MaxiSorp microtiter plates (Nunc, Roskilde, Denmark) were coated with $5 \mu \mathrm{g} / \mathrm{mL}$ unlabeled anti-mouse IgG (Southern Biotech) or IgA (PharMingen, C10-1) in PBS at $4{ }^{\circ} \mathrm{C}$ overnight. After washing the plates in PBS and blocking with $0.1 \%$ BSA/PBS, supernatants were diluted $1: 2$ in $0.1 \% B S A / P B S$ and added to wells. Standard (Purified mouse IgA K (M18-254) or purified mouse IgG1 K (MOPC-31C) from BD Pharmingen) was added in 7 wells of the first column with starting concentration $500 \mathrm{ng} / \mathrm{mL}$ and 2 -fold dilution. The final well was left as blank. Plates were kept at $4{ }^{\circ} \mathrm{C}$ overnight and following washing in PBS/ $0.5 \%$ Tween, the plates were incubated with AP-conjugated isotype-specific goat anti-mouse antibodies (Southern Biotechnology, Birmingham, AL). Plates were washed and the phosphatase substrate, p-nitrophenyl phosphatase (NPP) (Sigma) was added to each well. The reaction was read at $405 \mathrm{~nm}$ using a Titertek spectrophotometer (Flow Laboratories, Irvine, Scotland).

\section{Immunohistochemistry}

Mice were sacrificed, and the intestine, PP, SP, and MLN were embedded in TissueTek OCT compound and snap-frozen in liquid nitrogen. Frozen sections $(7 \mu \mathrm{m})$ were fixed in 100\% acetone and blocked with $5 \%$ normal horse serum in PBS for $15 \mathrm{~min}$. Antibodies used to stain sections were anti-GL7-eFlour 660 (GL7) from eBioscience, anti-CD4-BV785 (RMA4-5), anti-CD45.1-PE (A20), SA-PECy7, anti-lgA-biotin (C10.1), CD11C-BV421 (HL3), anti-B220-
V500 (RA3-6B2) obtained from BD Bioscience; anti-FOXP3-AF647 (150D), anti-Helios-BV421 (22F6), anti-CCR6-PE (29-2L17), anti-CD4PerCP (GK1.5) obtained from BioLegend. Microscopy was performed at the Centre for Cellular Imaging using the confocal Zeiss LSM 700 inv system and LSM software (Carl Zeiss, Oberkochen, Germany).

\section{Statistics}

Statistical analysis was performed using Graph Pad Prism7. Data are presented as mean \pm SEM or median with min to max using two way ANOVA with Tukey's multiple comparison test.

\section{ACKNOWLEDGEMENTS}

This study was supported by research funds from the Swedish Cancer Foundation, Lundberg Foundation, The Swedish Research Council, The Swedish Foundation for Strategic Research, The Knut and Alice Wallenberg's Foundation, EU projects in FP7 UniVacFlu and UNISEC. We thank Michel Nussenzweig for generously providing breeding pairs of the $\mathrm{B} 1-8^{\text {hi }}$ mouse strain. We thank Fredrik Bäckhed for providing germ-free mice. We also thank the Centre for Cellular Imaging and the Laboratory for Experimental Biomedicine facility at the University of Gothenburg.

\section{AUTHOR CONTRIBUTIONS}

Conception: I.G. and N.L.; research design: I.G. and N.L.; investigation: I.G., A.S., R.C., D. G.E. and N.L.; data analysis: I.G., A.S., D.G.E. and R.C.; resource assistance: R.C., A.S. and K.S.; writing-draft and editing: I.G., M.B. and N.L; and project administration: N.L.

\section{ADDITIONAL INFORMATION}

Competing interests: The authors declare no competing interests.

Publisher's note Springer Nature remains neutral with regard to jurisdictional claims in published maps and institutional affiliations.

\section{REFERENCES}

1. Fagarasan, S. \& Honjo, T. Intestinal IgA synthesis: regulation of front-line body defences. Nat. Rev. Immunol. 3, 63-72 (2003).

2. Komban, R. J. et al. Activated Peyer's patch $B$ cells sample antigen directly from $M$ cells in the subepithelial dome. Nat. Commun. 10, 2423 (2019).

3. Lycke, N. Y. \& Bemark, M. The regulation of gut mucosal IgA B-cell responses: recent developments. Mucosal. Immunol. 10, 1361-1374 (2017).

4. Kawanishi, H., Saltzman, L. E. \& Strober, W. Mechanisms regulating IgA classspecific immunoglobulin production in murine gut-associated lymphoid tissues. I. T cells derived from Peyer's patches that switch slgM B cells to slgA B cells in vitro. J. Exp. Med. 157, 433-450 (1983).

5. Cong, Y., Feng, T., Fujihashi, K., Schoeb, T. R. \& Elson, C. O. A dominant, coordinated T regulatory cell-IgA response to the intestinal microbiota. Proc. Natl Acad. Sci. USA 106, 19256-19261 (2009).

6. Hirota, K. et al. Plasticity of Th17 cells in Peyer's patches is responsible for the induction of T cell-dependent IgA responses. Nat. Immunol. 14, 372-379 (2013).

7. Sakaguchi, S., Sakaguchi, N., Asano, M., Itoh, M. \& Toda, M. Immunologic selftolerance maintained by activated $T$ cells expressing IL-2 receptor alpha-chains (CD25). Breakdown of a single mechanism of self-tolerance causes various autoimmune diseases. J. Immunol. 155, 1151-1164 (1995).

8. Tsuji, M. et al. Preferential generation of follicular B helper T cells from Foxp3+ T cells in gut Peyer's patches. Science 323, 1488-1492 (2009).

9. Vendetti, S. et al. Polyclonal Treg cells enhance the activity of a mucosal adjuvant. Immunol. Cell Biol. 88, 698-706 (2010).

10. Wang, L. et al. T regulatory cells and B cells cooperate to form a regulatory loop that maintains gut homeostasis and suppresses dextran sulfate sodium-induced colitis. Mucosal. Immunol. 8, 1297-1312 (2015).

11. Coffman, R. L., Lebman, D. A. \& Shrader, B. Transforming growth factor beta specifically enhances $\lg A$ production by lipopolysaccharide-stimulated murine $B$ lymphocytes. J. Exp. Med. 170, 1039-1044 (1989).

12. van Ginkel, F. W. et al. Partial IgA-deficiency with increased Th2-type cytokines in TGF- $\beta 1$ knockout mice. J. Immunol. 163, 1951-1957 (1999).

13. Cazac, B. B. \& Roes, J. TGF-beta receptor controls B cell responsiveness and induction of IgA in vivo. Immunity 13, 443-451 (2000).

14. Bauché, D. \& Marie, J. C. Transforming growth factor $\beta$ : a master regulator of the gut microbiota and immune cell interactions. Clin. Transl. Immunol. 6, e136 (2017). 
15. Cao, A. T. et al. Interleukin (IL)-21 promotes intestinal IgA response to microbiota. Mucosal. Immunol. 8, 1072-1082 (2015).

16. Hirota, K., Ahlfors, H., Duarte, J. H. \& Stockinger, B. Regulation and function of innate and adaptive interleukin-17-producing cells. EMBO Rep. 13, 113-120 (2012).

17. Kato, L. M., Kawamoto, S., Maruya, M. \& Fagarasan, S. Gut TFH and IgA: key players for regulation of bacterial communities and immune homeostasis. Immunol. Cell Biol. 92, 49-56 (2014).

18. Cao, A. T., Yao, S., Gong, B., Elson, C. O. \& Cong, Y. Th17 cells upregulate polymeric Ig receptor and intestinal $\lg A$ and contribute to intestinal homeostasis. $J$. Immunol. 189, 4666-4673 (2012).

19. DiPaolo, R. J., Glass, D. D., Bijwaard, K. E. \& Shevach, E. M. CD4+CD25+ T cells prevent the development of organ-specific autoimmune disease by inhibiting the differentiation of autoreactive effector T cells. J. Immunol. 175, 7135-7142 (2005).

20. Elkord, E. Thymus-derived, peripherally derived, and in vitro-induced T regulatory cells. Front Immunol. 5, 17 (2014).

21. Park, K. H., Seo, G. Y., Jang, Y. S. \& Kim, P. H. TGF- $\beta$ and BAFF derived from CD4 $+\mathrm{CD} 25+$ Foxp3 + T cells mediate mouse IgA isotype switching. Genes Genomics. 34, 619-625 (2012).

22. Gros, M. J., Naquet, P. \& Guinamard, R. R. Cell intrinsic TGF-beta 1 regulation of $B$ cells. J. Immunol. 180(Jun), 8153-8158 (2008).

23. Reboldi, A. et al. IgA production requires $B$ cell interaction with subepithelia dendritic cells in Peyer's patches. Science 352, aaf4822 (2016).

24. Travis, M. A. \& Sheppard, D. TGF- $\beta$ activation and function in immunity. Annu Rev. Immunol. 32, 51-82 (2014).

25. Nagashima, K. et al. Identification of subepithelial mesenchymal cells that induce IgA and diversify gut microbiota. Nat. Immunol. 18, 675-682 (2017).

26. Rios, D. et al. Antigen sampling by intestinal $M$ cells is the principal pathway initiating mucosal $\lg \mathrm{A}$ production to commensal enteric bacteria. Mucosal. Immunol. 9, 907-916 (2016).

27. Park, M. H., Park, S. R., Lee, M. R., Kim, Y. H. \& Kim, P. H. Retinoic acid induces expression of Ig germ line a transcript, an IgA isotype switching indicative, through retinoic acid receptor. Genes Genome 33, 83-88 (2011).

28. Rickert, R. C., Jellusova, J. \& Miletic, A. V. Signaling by the tumor necrosis factor receptor superfamily in B-cell biology and disease. Immunol. Rev. 244, 115-133 (2011).

29. Castigli, E. et al. Impaired IgA class switching in APRIL-deficient mice. Proc. Nat/ Acad. Sci. USA 101, 3903-3908 (2004).

30. Lycke, N. Recent progress in mucosal vaccine development: potential and limitations. Nat. Rev. Immunol. 12, 592-605 (2012).

31. Murphy, K. M., Heimberger, A. B. \& Loh, D. Y. Induction by antigen of intrathymic apoptosis of CD4+CD8+TCRlo thymocytes in vivo. Science 250, 1720-1723 (1990).

32. Robertson, J. M., Jensen, P. E. \& Evavold, B. D. DO11.10 and OT-II T cells recognize a C-terminal ovalbumin 323-339 epitope. J. Immunol. 164, 4706-4712 (2000).

33. Lavelle, E. \& O'Hagan, D. Delivery systems and adjuvants for oral vaccines. Expert Opin. Drug Deliv. 3, 747-762 (2006).

34. Lycke, N. \& Lebrero-Fernández, C. ADP-ribosylating enterotoxins as vaccine adjuvants. Curr. Opin. Pharmacol. 41, 42-51 (2018).

35. Pantelouris, E. M. Athymic development in the mouse. Differentiation 1, 437-450 (1973).

36. Rezende, R. M. \& Weiner, H. L. History and mechanisms of oral tolerance. Semin Immunol. 30, 3-11 (2017).

37. Worbs, T. et al. Oral tolerance originates in the intestinal immune system and relies on antigen carriage by dendritic cells. J. Exp. Med. 203, 519-527 (2006).

38. Karlsson, M. R., Johansen, F. E., Kahu, H., Macpherson, A. \& Brandtzaeg, P. Hypersensitivity and oral tolerance in the absence of a secretory immune system. Allergy 65, 561-570 (2010).

39. Pabst, O. \& Mowat, A. M. Oral tolerance to food protein. Mucosal. Immunol. 5, 232-239 (2012)
40. Shih, T. A., Roederer, M. \& Nussenzweig, M. C. Role of antigen receptor affinity in T cell-independent antibody responses in vivo. Nat. Immunol. 3, 399-406 (2002).

41. Moreau, M., Ducluzeau, R., Guy-Grand, D. \& Muller, M. Increase in the population of duodenal immunoglobulin A plasmocytes in axenic mice associated with different living or dead bacterial strains of intestinal origin. Infect. Immun. 21 532-539 (1978).

42. Lee, W. T., Cole-Calkins, J. \& Street, N. E. Memory T cell development in the absence of specific antigen priming. J. Immunol. 157, 5300-5307 (1996).

43. Zhou, P. et al. Expression of dual TCR on DO11.10 T cells allows for ovalbumininduced oral tolerance to prevent $\mathrm{T}$ cell-mediated colitis directed against unrelated enteric bacterial antigens. J. Immunol. 172(Feb), 1515-1523 (2004).

44. Hurst, S. D., Sitterding, S. M., Ji, S. \& Barrett, T. A. Functional differentiation of T cells in the intestine of T cell receptor transgenic mice. Proc. Natl Acad. Sci. USA 94, 3920-3925 (1997)

45. Bergqvist, $\mathrm{P}$. et al. Re-utilization of germinal centers in multiple Peyer's patches results in highly synchronized, oligoclonal, and affinity-matured gut $\lg A$ responses. Mucosal. Immunol. 6, 122-135 (2013).

46. Jinnin, M., Ihn, H. \& Tamaki, K. Characterization of SIS3, a novel specific inhibitor of Smad3, and its effect on transforming growth factor-beta1-induced extracellular matrix expression. Mol. Pharmacol. 69, 597-607 (2006). Epub 2005 Nov 15.

47. Moon, J. J. et al. Naive CD4(+) T cell frequency varies for different epitopes and predicts repertoire diversity and response magnitude. Immunity 27, 203-213 (2007).

48. Takahashi, $H$. et al. TGF- $\beta$ and retinoic acid induce the microRNA miR-10a, which targets $\mathrm{BCl}-6$ and constrains the plasticity of helper T cells. Nat. Immunol. 13, 587-595 (2012).

49. Ellmeier, W. Molecular control of CD4(+) T cell lineage plasticity and integrity. Int Immunopharmacol. 28, 813-817 (2015).

50. Zhou, L., Chong, M. M. \& Littman, D. R. Plasticity of CD4+ T cell lineage differentiation. Immunity 30, 646-655 (2009).

51. Balakrishnan, A. \& Morris, G. P. The highly alloreactive nature of dual TCR T cells. Curr. Opin. Organ Transplant. 21, 22-28 (2016).

52. Schuldt, N. J. et al. Cutting edge: dual TCRa expression poses an autoimmune hazard by limiting regulatory T cell generation. J. Immunol. 199, 33-38 (2017).

53. Bergqvist, P., Stensson, A., Lycke, N. Y. \& Bemark, M. T cell-independent IgA class switch recombination is restricted to the GALT and occurs prior to manifest germinal center formation. J. Immunol. 184, 3545-3553 (2010).

54. Ashcroft, G. S. et al. Mice lacking Smad3 show accelerated wound healing and an impaired local inflammatory response. Nat. Cell Biol. 1, 260-266 (1999).

55. Klein, J. et al. B cell-specific deficiency for Smad2 in vivo leads to defects in TGFbeta-directed IgA switching and changes in B cell fate. J. Immunol. 176, 2389-2396 (2006).

56. Frick, C. L., Yarka, C., Nunns, H. \& Goentoro, L. Sensing relative signal in the Tgf- $\beta$ / Smad pathway. Proc. Natl Acad. Sci. USA 114, E2975-E2982 (2017).

57. Luo, J., Lin, A. H., Masliah, E. \& Wyss-Coray, T. Bioluminescence imaging of Smad signaling in living mice shows correlation with excitotoxic neurodegeneration. Proc. Natl Acad. Sci. USA 103, 18326-18331 (2006).

58. Rubtsov, Y. et al. Stability of the regulatory T cell lineage in vivo. Science 329, 1667-1671 (2010).

59. Laidlaw, B. J. et al. Interleukin-10 from CD4+ follicular regulatory $T$ cells promotes the germinal center response. Sci. Immunol. 20, 2 (2017).

60. Sage, P. T. \& Sharpe, A. H. T follicular regulatory cells in the regulation of B cell responses. Trends Immunol. 36, 410-418 (2015).

61. Sayin, I. et al. Spatial distribution and function of $T$ follicular regulatory cells in human lymph nodes. J. Exp. Med. 215, 1531-1542 (2018).

62. Lefrançois, L. \& Lycke, N. Isolation of mouse small intestinal intraepithelial lymphocytes, Peyer's patch, and lamina propria cells. Curr. Protoc. Immunol. Unit3, 19 (2001). Chapter 3.

63. Lycke, N. \& Coico, R. ELISPOT assay for measurement of antigen-specific and polyclonal antibody responses. Curr. Protoc. Immunol. 108, 7.14.1-10 (2015). 